Article

\title{
Liver Injury and Elevated FIB-4 Define a High-Risk Group in Patients with COVID-19
}

\author{
Dana Crisan 1,2, Lucretia Avram 1,2,*iD, Cristiana Grapa 1,*, Alexandra Dragan ${ }^{1}$, Dan Radulescu ${ }^{1,2}$, Sorin Crisan ${ }^{1,2}$, \\ Alin Grosu 1,2, Valentin Militaru 1,2, Elena Buzdugan 1,2, Laurentiu Stoicescu 1,2, Liliana Radulescu 1,2, \\ Felix Ciovicescu 1,2, Delia Bunea Jivanescu 1,2, Oana Mocan 1,2, Bogdan Micu 1,3, Valer Donca 1,2, \\ Luminita Marinescu 1,2, Antonia Macarie 1,2, Marina Rosu ${ }^{1,2}$, Andrada Nemes ${ }^{4}$ and Rares Craciun ${ }^{1}$ (D)
}

1 Faculty of Medicine, “Iuliu Hatieganu” University of Medicine and Pharmacy, 400000 Cluj-Napoca, Romania; crisan.dc@gmail.com (D.C.); alexandra.dragan@elearn.umfcluj.ro (A.D.); dan.radulescu@umfcluj.ro (D.R.); Sorin.Crisan@umfcluj.ro (S.C.); alin.grosu@umfcluj.ro (A.G.); valentin.militaru@umfcluj.ro (V.M.); cristina.buzdugan@umfcluj.ro (E.B.); laurentiu.stoicescu@umfcluj.ro (L.S.); lili_m_radulescu@yahoo.com (L.R.); felix.ciovicescu@umfcluj.ro (F.C.); bunea.delia@umfcluj.ro (D.B.J.); oana.mocan@umfcluj.ro (O.M.); micu.vasile@umfcluj.ro (B.M.); ioan.donca@umfcluj.ro (V.D.); pasca.luminita@umfcluj.ro (L.M.); macarie.antonia@umfcluj.ro (A.M.); ROSU.MARINA@elearn.umfcluj.ro (M.R.); craciun.rares.calin@elearn.umfcluj.ro (R.C.)

2 Department of Internal Medicine, Clinical Municipal Hospital Cluj-Napoca, 400139 Cluj-Napoca, Romania 3 Department of General Surgery, Clinical Municipal Hospital Cluj-Napoca, 400139 Cluj-Napoca, Romania

4 Intensive Care Unit I, Cluj County Emergency Hospital, 400006 Cluj-Napoca, Romania; nemes.andrada.raluca@elearn.umfcluj.ro

* Correspondence: avram.lucretia9@gmail.com (L.A.); cristianagrapa@yahoo.com (C.G.)

check for updates

Citation: Crisan, D.; Avram, L.; Grapa, C.; Dragan, A.; Radulescu, D.; Crisan, S.; Grosu, A.; Militaru, V.; Buzdugan, E.; Stoicescu, L.; et al. Liver Injury and Elevated FIB-4 Define a High-Risk Group in Patients with COVID-19. J. Clin. Med. 2022, 11 153. https://doi.org/10.3390/ jcm11010153

Academic Editor: Maria Guarino

Received: 29 November 2021

Accepted: 24 December 2021

Published: 28 December 2021

Publisher's Note: MDPI stays neutral with regard to jurisdictional claims in published maps and institutional affiliations.

Copyright: (C) 2021 by the authors. Licensee MDPI, Basel, Switzerland. This article is an open access article distributed under the terms and conditions of the Creative Commons Attribution (CC BY) license (https:// creativecommons.org/licenses/by/ $4.0 /)$.

\begin{abstract}
Liver involvement in Coronavirus Disease 2019 (COVID-19) has been widely documented. However, data regarding liver-related prognosis are scarce and heterogeneous. The current study aims to evaluate the role of abnormal liver tests and incidental elevations of non-invasive fibrosis estimators on the prognosis of hospitalized COVID-19 patients. We conducted a retrospective cohort study to investigate the impact of elevated liver tests, non-invasive fibrosis estimators (the Fibrosis-4 (FIB-4), Forns, APRI scores, and aspartate aminotransferase/alanine aminotransferase (AST/ALT) ratio), and the presence of computed tomography (CT)-documented liver steatosis on mortality in patients with moderate and severe COVID-19, with no prior liver disease history. A total of 370 consecutive patients were included, of which 289 patients (72.9\%) had abnormal liver biochemistry on admission. Non-survivors had significantly higher FIB-4, Forns, APRI scores, and a higher AST/ALT ratio. On multivariate analysis, severe FIB-4 (exceeding 3.25) and elevated AST were independently associated with mortality. Severe FIB-4 had an area under the receiver operating characteristic (AUROC) of 0.73 for predicting survival. The presence of steatosis was not associated with a worse outcome. Patients with abnormal liver biochemistry on arrival might be susceptible to a worse disease outcome. An FIB-4 score above the threshold of 3.25, suggestive of the presence of fibrosis, is associated with higher mortality in hospitalized COVID-19 patients.
\end{abstract}

Keywords: COVID-19; liver; FIB-4; mortality

\section{Introduction}

The systemic nature of Severe Acute Respiratory Syndrome Coronavirus 2 (SARS-CoV2) infection has been well documented since the COVID-19 pandemic ground the world to a halt in early 2020 [1]. In the subsequent months, a collective effort emerged, as numerous teams reported their experience in an attempt to sketch out the disease's physiology, natural history, and potential therapeutic targets. In the meantime, the scientific community has achieved the improbable task of quickly developing multiple safe and effective vaccines. Yet, almost two years after being initially reported in Wuhan, the pandemic flame is far from being extinguished. Largely due to unequal vaccine availability, 
widespread anti-vaccination movements, and the emergence of new viral variants, the pandemic remains unpredictable. Thus, all additional information brought to light becomes extremely valuable.

The independent outcome predictor status of cardiovascular disease, metabolic syndrome, and diabetes mellitus on the course of COVID-19 was observed early and widely discussed [2-4]. Moreover, there are multiple other high-risk groups that are becoming increasingly better defined, such as immunocompromised patients (either transplant recipients or other groups on immunosuppressive medication) or patients with ongoing malignancy [5-7] However, the involvement of the liver either in the pathophysiology of COVID-19 or as an underlying risk factor was initially inconspicuous, even if approximately half of the patients had abnormal liver tests on admission [8]. In the latter months of 2020 and early 2021, however, multiple reports have been published on the role of abnormal liver workup, especially in patients with undocumented liver disease, fibrosis, and/or quiescent Metabolic Associated Fatty Liver Disease (MAFLD) and undiagnosed Non-Alcoholic Steatohepatitis (NASH).

The largest part of liver-related clinical research (excluding portal hypertension) in COVID-19 consists of two subtopics: the relevance of liver test abnormalities during hospitalization and the role of MAFLD as an outcome predictor. As per se abnormal tests have little relevance out of context, the first matter converged towards the role of non-invasive scores in assessing various outcomes, such as disease severity, hospitalization, and, ultimately, mortality. Among the most useful tools, the FIB-4 score has gained the most traction. Initially developed as a simple four-variable tool to estimate fibrosis in $\mathrm{HCV} / \mathrm{HIV}$ co-infected patients [9] and further validated on a wider range of etiologies, including MAFLD [10], FIB-4 has hinted towards applicability in COVID-19, as some recently published papers suggest [11-14]. Along with FIB-4, there are multiple other easy to use non-invasive scores, such as FORNS [15], APRI [16], and the AST to ALT ratio [17]. These scores have extensive validation in other liver disease etiologies and might extend their applicability in the setting of COVID-19.

On the other hand, the role of MAFLD has not been clearly established. MAFLD is a common finding in patients with metabolic syndrome and/or diabetes mellitus, which are clear risk factors for a severe COVID-19 disease course and mortality. Classifying MAFLD patients as high-risk for a poor outcome can be grounded on the assumption that an intricate interrelation between obesity, vitamin D deficiency, and a chronic low-grade pro-inflammatory status leads to an ill-adapted immune response [18]. However, while MAFLD plus fibrosis (highly suggestive of NASH) appears to be associated with a worse outcome $[13,19]$, there is conflicting data with regards to the role of quiescent MAFLD or incidental findings of steatosis in predicting definite endpoints [13,20,21].

Moreover, in the reorganized hospital settings, the accessibility of high-precision diagnostic tools has substantially decreased during the pandemic. Techniques such as transient elastography or high-performance ultrasound machines with pre-installed shearwave elastography and steatosis assessment applications remained underused, as care moved closer to the bedside. This inconvenience further places the burden on quick, low-resource-consuming tools to provide essential diagnostic and prognostic data.

Aims

Our current study had the major objective of establishing the role of abnormal liver tests and incidental findings of fibrosis on admission, assessed by FIB-4, FORNS, APRI, AST / ALT in predicting disease outcome in patients with undocumented pre-existing liver disease hospitalized for SARS-CoV2 infection. A secondary objective, in tight conjunction with the first one, was to evaluate the role of incidental findings of liver steatosis, diagnosed on admission at the thoracic CT scan, on disease course, intensive care unit admission, and mortality. 


\section{Materials and Methods}

\subsection{Study Design and Participants}

The current research was designed as an observational, retrospective, longitudinal study. Patient enrollment took place in a single tertiary-care hospital, which has been transformed into a regional COVID-19-dedicated center. A consecutive series of patients was included between January 2021 and April 2021. All patients tested positive for SARS-CoV-2 RNA following a nasopharyngeal swab analyzed by real-time reversetranscriptase polymerase chain reaction (rRT-PCR). None of the patients were vaccinated against SARS-CoV-2.

Per regional protocol, asymptomatic and mildly symptomatic patients with no additional risk factors (young, no documented associated conditions, negative thoracic CT scan) were not hospitalized, and thus, not included in our analysis. The rationale for not including such patients in our study was based on the intention to avoid skewing the dataset towards the less severe end of the disease spectrum, to avoid overestimating the effect size of the predictive analysis. Patients with previously diagnosed liver disease were excluded: infection with hepatitis B virus (HBV), untreated infection with hepatitis $\mathrm{C}$ virus (HCV), treated $\mathrm{HCV}$ infection with documented fibrosis, alcoholic liver disease or alcohol misuse disorder, biopsy-proven NASH, autoimmune hepatitis, primary biliary cholangitis, primary sclerosing cholangitis, hemochromatosis, Wilson's disease, vascular liver disease, documented cirrhosis or clinically significant portal hypertension of any etiology, with or without any prior decompensation. The risk for drug-induced liver injury was assessed by analyzing chronic and recent medication on arrival. When found, patients on the drugs most frequently associated with liver injury were excluded. Patients with previously diagnosed myopathies, recent trauma, hematological conditions, and transplant recipients were also excluded.

\subsection{Baseline Evaluation}

Demographic data, as well as a comprehensive laboratory workup, were performed either in the emergency department or during day 1 of hospitalization. The laboratory workup consisted of a full blood count, inflammatory syndrome assessment (C-reactive protein (CRP), procalcitonin, D-dimer, fibrinogen, lactate dehydrogenase (LDH) and ferritin), liver function tests (aspartate aminotransferase (AST), and alanyl aminotransferase (ALT), total and direct bilirubin, GGT, alkaline phosphatase (ALP), albumin), coagulation (prothrombin time, international normalized ratio (INR), activated partial thromboplastin time (aPTT)), kidney function (urea, creatinine, electrolytes), and metabolic profile (fasting blood glucose, triglyceride levels, total cholesterol, HDL and LDL cholesterol levels, total protein levels). All patients with liver test abnormalities were screened for HBV and HCV infection, using HBs antigen and HCV antibodies, respectively.

A non-contrast thoracic computed tomography scan was performed on admission. The total severity score (TSS) was used to assess COVID-19 lung involvement, as described by Li K. et al. [22]. Patients were classified as having a severe form of the disease if the TSS score exceeded 8. Hepatic steatosis was assessed using a previously validated protocol [13]. CT scans were interpreted by a single radiologist. The diagnosis of liver steatosis was based upon two major criteria: an attenuation coefficient of less than 40 Hounsfield Units (HU) in a $20 \mathrm{~cm}^{2}$ area in segments VII and VIII and an attenuation coefficient above $10 \mathrm{HU}$ in a $5 \mathrm{~cm}^{2}$ area in the splenic parenchyma compared to the previously described area in the liver. A liver/spleen ratio cut-off value of 0.7 was used to discriminate patients with severe steatosis.

\subsection{Non-Invasive Scores}

All scores were calculated using the laboratory data obtained on admission, as originally described. Cut-off values were used to define high-risk groups for the presence of advanced steatosis, according to the initial validation studies. To account for the low specificity of FIB-4 in elderly patients (age group > 65), a cut-off of 2 was used, as previously 
described [23]. Calculation formulas and cut-off values are depicted in the table below (Table 1).

Table 1. Non-invasive fibrosis scores.

\begin{tabular}{|c|c|c|c|}
\hline Non-Invasive Score & As Originally Described in: & Formula & $\begin{array}{l}\text { Cut-Off Values for High } \\
\text { Risk of Advanced Fibrosis }\end{array}$ \\
\hline FIB-4 & Sterling R. et al. [9] & $\begin{array}{c}\text { Age }(\text { years }) \times \text { AST }(\text { IU } / \text { L }) / \text { platelet } \\
\text { count }\left(\times 10^{9} / \mathrm{L}\right) \times \sqrt{ } \text { ALT }(\mathrm{IU} / \mathrm{L})\end{array}$ & $\begin{array}{l}3.25 \text { if age }<65 \\
2.0 \text { if age } \geq 65\end{array}$ \\
\hline AST/ALT ratio & Giannini E. et al. [17] & AST/ALT & 0.8 \\
\hline Forns score & Wai C. et al. [15] & $\begin{array}{c}7.811-3.131 \times \ln (\text { platelet count })+ \\
0.781 \times \ln (\mathrm{GGT})+3.467 \times \ln (\text { age }) \\
\quad-0.014 \times(\text { total cholesterol })\end{array}$ & 6.9 \\
\hline APRI score & Lin Z. et al. [16] & $\begin{array}{c}\text { AST/upper limit of } \\
\text { normal)/platelet count }(\text { expressed } \\
\left.\text { as platelets } \times 10^{9} / \mathrm{L}\right) \times 100\end{array}$ & 1 \\
\hline
\end{tabular}

\subsection{Follow-Up}

Patients were followed during their hospitalization, recording total hospital stay, admission to the intensive care unit (ICU), and death.

\subsection{Ethical Considerations}

By design, the study complies with all current ethical considerations. The protocol was approved by the Institutional Ethics Committee of the Clinical Municipal Hospital in Cluj-Napoca. Informed written consent was obtained from each patient included in the study and the study protocol was developed under the ethical guidelines of the modified 1975 Declaration of Helsinki.

\subsection{Statistical Analysis}

Continuous variables were expressed as either mean \pm standard deviation (SD) or median and 95\% confidence interval (CI), for normal and non-normal distributions, respectively. Student's $t$-test was used for the comparison of normally distributed variables, while the Mann-Whitney U test was used for non-normal variables. The chi-square test was used for categorical variables. Multivariate analysis was designed to minimize model overfitting. Kaplan-Meyer curves with the log-rank test were used for survival analysis. The threshold for statistical significance was set at 0.05 . The discriminative abilities of different variables were analyzed using the receiver operating characteristic (ROC) curve. Statistical analysis was performed by a certified biomedical statistician using SPSS software, version 28.0 (SPSS Inc., Chicago, IL, USA).

\section{Results}

\subsection{Baseline Characteristics}

Our cohort consisted of 370 patients with confirmed SARS-CoV-2 infection. The median age was 65.5 years old (58.11-69.3), with a slight male predominance $(n=220$, $59.49 \%)$. More than half of the patients (51.6\%) had severe COVID-19 according to the TSS score (TSS $>8)$. The most common co-morbid condition was arterial hypertension $(n=217$, $58.8 \%)$, followed by type 2 diabetes mellitus $(n=90,24.3 \%)$. None of the patients had a prior history of chronic liver disease.

Throughout hospital stay, 289 (72.94\%) patients developed a form of liver injury, defined by AST and ALT elevations, and 165 (50.92\%) developed a cholestatic pattern with both ALP and GGT elevated. On admission, median AST and ALT levels were 47.5 (47.26-93.33) and 38 (33.24-77.17), while the peak values during hospitalization were 69.5 (65.82-130.28) and 86.5 (84.66-220.94), respectively. As assessed by analyzing liver parenchyma densities on the index CT scan, the prevalence of liver steatosis was $39.5 \%$ 
$(n=145)$. Regarding the non-invasive tests used for the diagnosis and staging of liver fibrosis, FIB-4 had a median value of 2.6 (2.1-4.71), AST / ALT 1.51 (1.26-1.84), APRI 0.42 $(0.4-1.13)$ and Forns 6.61 (5.78-7.11). Severe fibrosis according to FIB-4 was found in 109 $(29.5 \%)$ patients and $156(42.2 \%)$ after adjusting the score for age $>65$, according to guideline recommendations. The baseline characteristics of our cohort are summarized in Table 2.

Table 2. Baseline characteristics.

\begin{tabular}{|c|c|}
\hline Variable & Median (Confidence Interval)/No (\%) \\
\hline Age (years) & $65.5(58.11-69.3)$ \\
\hline Gender $(\mathrm{M}, \%)$ & $220(59.45 \%)$ \\
\hline \multicolumn{2}{|l|}{ Biological parameters } \\
\hline Hemoglobin $(\mathrm{g} / \mathrm{dL})$ & $13.05(11.54-13.96)$ \\
\hline White blood cells $\left(\times 10^{9} / \mathrm{L}\right)$ & $7.05(6.96-9.43)$ \\
\hline Lymphocytes $\left(\times 10^{9} / \mathrm{L}\right)$ & $1.09(0.93-1.36)$ \\
\hline Neutrophils $\left(\times 10^{9} / \mathrm{L}\right)$ & $5.42(5.52-7.57)$ \\
\hline Platelets $\left(\times 10^{9} / \mathrm{L}\right)$ & $207.5(193.04-266.21)$ \\
\hline Random blood glucose (on admission, $\mathrm{mg} / \mathrm{dL}$ ) & $126(119.94-172.64)$ \\
\hline C-reactive protein $(\mathrm{mg} / \mathrm{L})$ & $139.5(104.68-169.61)$ \\
\hline Procalcitonin (ng/mL) & $0.14(-0.22-1.47)$ \\
\hline Fibrinogen $(\mathrm{mg} / \mathrm{dL})$ & 715.65 (682.69-941.94) \\
\hline D-dimers (mg/dL) & $529.45(489.79-1333.77)$ \\
\hline Ferritin $(\mathrm{mg} / \mathrm{dL})$ & $824.1(763.21-1808.79)$ \\
\hline Lactate dehydrogenase (U/L) & $697.5(515.86-840.3)$ \\
\hline Total cholesterol (mg/dL) & 155.5 (142.65-177.42) \\
\hline Triglycerides (mg/dL) & $144(130.79-198.37)$ \\
\hline HDL-cholesterol (mg/dL) & $32.5(31.1-41.4)$ \\
\hline Creatinine $(\mathrm{mg} / \mathrm{dL})$ & $1.02(0.76-2.33)$ \\
\hline Urea $(\mathrm{mg} / \mathrm{dL})$ & $43(39.04-64.99)$ \\
\hline $\mathrm{Na}(\mathrm{mmol} / \mathrm{L})$ & $139(136.36-139.73)$ \\
\hline $\mathrm{K}(\mathrm{mmol} / \mathrm{L})$ & $4.31(4.23-4.69)$ \\
\hline Creatinine clearance (MDRD) $\left(\mathrm{mL} / \mathrm{min} / 1.73 \mathrm{~m}^{2}\right)$ & $64.47(52.26-78.74)$ \\
\hline INR & $1.07(1-1.59)$ \\
\hline Prothrombin Time (s) & $17.85(16.05-24.7)$ \\
\hline \multicolumn{2}{|l|}{ Liver tests } \\
\hline AST (U/L) & $47.5(47.26-93.33)$ \\
\hline $\operatorname{ALT}(\mathrm{U} / \mathrm{L})$ & $38(33.24-77.17)$ \\
\hline AST and ALT elevations $(\mathrm{N}, \%)$ & $289(72.94 \%)$ \\
\hline $\operatorname{ALP}(\mathrm{U} / \mathrm{L})$ & $67.5(63.17-92.24)$ \\
\hline GGT (U/L) & $91(71.18-134.9)$ \\
\hline ALP and GGT elevations (N, \%) & $165(50.92 \%)$ \\
\hline Bilirubin (mg/dL) & $0.45(0.41-0.66)$ \\
\hline Fibrosis scores & \\
\hline
\end{tabular}


Table 2. Cont.

\begin{tabular}{|c|c|}
\hline Variable & Median (Confidence Interval)/No (\%) \\
\hline AST/ALT value & $1.51(1.26-1.84)$ \\
\hline Severe AST/ALT & $163(44.1 \%)$ \\
\hline APRI value & $0.42(0.4-1.13)$ \\
\hline Severe APRI & $88(23.8 \%)$ \\
\hline FIB-4 value & $2.6(2.1-4.71)$ \\
\hline Severe FIB-4 & $109(29.5 \%)$ \\
\hline Age adjusted severe FIB- 4 & $156(42.2 \%)$ \\
\hline Forns value & $6.61(5.78-7.11)$ \\
\hline Severe Forns & $199(53.8 \%)$ \\
\hline \multicolumn{2}{|l|}{ Disease severity and personal history } \\
\hline TSS start & $11.5(10.44-13.31)$ \\
\hline TSS maxim & $11.5(10.58-13.75)$ \\
\hline Severe COVID * & $191(51.6 \%)$ \\
\hline Liver steatosis on CT & $146(39.5 \%)$ \\
\hline Arterial hypertension & $217(58.6 \%)$ \\
\hline Ischemic heart disease & $62(16.8 \%)$ \\
\hline Atrial fibrillation & $38(10.3 \%)$ \\
\hline Heart failure & $42(11.4 \%)$ \\
\hline Chronic obstructive pulmonary disease & $34(9.2 \%)$ \\
\hline Stroke & $25(6.8 \%)$ \\
\hline Chronic kidney disease & $44(11.9 \%)$ \\
\hline Type 2 diabetes mellitus & $90(24.3 \%)$ \\
\hline Neoplasia & $27(7.3 \%)$ \\
\hline \multicolumn{2}{|c|}{$\begin{array}{l}\text { Abbreviations: ALT—alanine aminotransferase; AST—aspartate aminotransferase; ALP-alkaline phosphatase; } \\
\text { GGT-gamma glutamyl transferase; CRP-C reactive protein; LDH-lactate dehydrogenase; TG-triglycerides; } \\
\text { LDL-low density lipoproteins; HDL—high density lipoproteins; INR-international normalized ratio; PT- } \\
\text { prothrombin time; CT-computer tomography; COPD—Chronic obstructive pulmonary disease; CVA- } \\
\text { cerebrovascular accident; T2DM-type } 2 \text { diabetes mellitus; }{ }^{*} \text { Severe COVID according to TSS scoring (TSS > 8). } \\
\text { Variables are shown as median ( } 95 \% \text { confidence interval). }\end{array}$} \\
\hline
\end{tabular}

\subsection{Univariate Outcome Analysis}

A univariate analysis was performed to assess the variables associated with in-hospital mortality. Of the 370 total patients, $43(11.6 \%)$ have died during hospitalization. Nonsurvivors had a significantly higher TSS score, were older, had a more pronounced proinflammatory status (as derived from higher levels of CRP, procalcitonin, ferritin, fibrinogen, D-dimers, and LDH), and had worse kidney function. Non-survivors had significantly lower platelet counts. Among liver function tests, on admission AST and peak AST levels were significantly higher in the latter group. According to CT scans, $20(46.51 \%)$ patients in the non-survivor group and $126(38.53 \%)$ in the survivor group were diagnosed with liver steatosis, yet with no statistical difference. Concerning the fibrosis scores, FIB-4 $(p<0.005)$ and the prevalence of severe fibrosis (as assessed by FIB-4 cut-offs) were significantly higher in the non-survivor group $(p=0.04)$. All the other scores, namely AST / ALT $(p<0.001)$, APRI $(p=0.009)$, and Forns $(p=0.001)$ were also significantly associated with a worse outcome, with higher values in non-survivors. The differences between the two groups are summarized in Table 3. 
Table 3. Univariate comparison between survivors and non-survivors.

\begin{tabular}{|c|c|c|c|}
\hline Variable & Survivors $(n=327,88.4 \%)$ & Non-Survivors $(n=43,11.6 \%)$ & $p$ \\
\hline Age (years) & $62(55.4-67.5)$ & $73(60.53-89.47)$ & $<0.001$ \\
\hline Gender $(\mathrm{M}, \%)$ & $190(86.36 \%)$ & $30(69.76 \%)$ & 0.096 \\
\hline \multicolumn{4}{|l|}{ Biological parameters } \\
\hline Hemoglobin (g/dL) & $13.05(11.24-14.04)$ & $13(9.5-17.09)$ & 0.006 \\
\hline White blood cells $\left(\times 10^{9} / \mathrm{L}\right)$ & $7.45(6.96-9.74)$ & $6.22(2.59-12.35)$ & 0.23 \\
\hline Lymphocytes $\left(\times 10^{9} / \mathrm{L}\right)$ & $1.09(0.94-1.40)$ & $0.85(0.00-2.05)$ & $<0.001$ \\
\hline Neutrophils $\left(\times 10^{9} / \mathrm{L}\right)$ & $5.57(5.47-7.79)$ & $5.42(2.19-10)$ & 0.017 \\
\hline Platelets $\left(\times 10^{9} / \mathrm{L}\right)$ & $231(206-285)$ & $153.5(72.98-227.52)$ & 0.035 \\
\hline Random blood sugar (on admission, mg/dL) & $125(116.01-178.69)$ & $128(81.31-200.69)$ & 0.026 \\
\hline $\mathrm{C}$ reactive protein $(\mathrm{mg} / \mathrm{L})$ & $132.29(92.6-169.65)$ & $169.5(118.35-216.14)$ & $<0.001$ \\
\hline Procalcitonin (ng/mL) & $0.13(0.01-1.68)$ & $0.25(0.01-1.3)$ & 0.001 \\
\hline Fibrinogen $(\mathrm{mg} / \mathrm{dL})$ & $746.2(677.67-989.72)$ & $710.15(677.65-733.19)$ & 0.015 \\
\hline D-dimers (mg/dL) & $529.45(461.6-934.43)$ & $1871.15(-1285.41-5246.66)$ & 0.04 \\
\hline $\mathrm{LDH}(\mathrm{U} / \mathrm{L})$ & $627(481.46-874.93)$ & $698.5(500.33-854.66)$ & $<0.001$ \\
\hline Ferritin (mg/dL) & $742.75(696.38-1956.5)$ & $1073.5(358.78-1808.81)$ & 0.008 \\
\hline Cholesterol (mg/dL) & $159.5(142.76-184.04)$ & 140.5 (119.17-167.32) & 0.001 \\
\hline $\mathrm{TG}(\mathrm{mg} / \mathrm{dL})$ & 144 (128.5-208.69) & $140.5(74-215)$ & 0.557 \\
\hline $\mathrm{HDL}(\mathrm{mg} / \mathrm{dL})$ & $31.5(29.23-40.87)$ & $38.5(26.06-58.44)$ & 0.881 \\
\hline $\mathrm{LDL}(\mathrm{mg} / \mathrm{dL})$ & $100(83.74-121.96)$ & $71(58.54-85.96)$ & 0.014 \\
\hline Creatinine (mg/dL) & $0.96(0.53-2.36)$ & $1.75(0.07-4.23)$ & $<0.001$ \\
\hline Urea (mg/dL) & $38.5(34.76-51.98)$ & $90.5(22.21-168.28)$ & $<0.001$ \\
\hline $\mathrm{Na}(\mathrm{mmol} / \mathrm{L})$ & $139(135.93-139.77)$ & $138.5(133.05-144.95)$ & 0.115 \\
\hline $\mathrm{K}(\mathrm{mmol} / \mathrm{L})$ & $4.41(4.25-4.72)$ & $3.95(3.01-5.67)$ & 0.008 \\
\hline Creatinine clearance $(\mathrm{MDRD})\left(\mathrm{mL} / \mathrm{min} / 1.73 \mathrm{~m}^{2}\right)$ & $67.62(55.16-83.85)$ & $40.39(3.38-94.37)$ & $<0.001$ \\
\hline INR & $1.07(0.96-1.68)$ & $1.14(0.92-1.37)$ & 0.004 \\
\hline Prothrombin Time (s) & $17.85(15.18-25.58)$ & $18.75(12.73-27.96)$ & 0.008 \\
\hline \multicolumn{4}{|l|}{ Liver tests } \\
\hline AST (U/L) & $51.02(46.97-55.07)$ & $64.55(49.76-79.35)$ & 0.04 \\
\hline $\operatorname{ALT}(\mathrm{U} / \mathrm{L})$ & $50.45(45.05-55.86)$ & $38.95(27.58-50.31)$ & 0.13 \\
\hline $\operatorname{ALP}(\mathrm{U} / \mathrm{L})$ & $71.5(65.64-87.76)$ & $52.5(-35.33-200.83)$ & 0.212 \\
\hline GGT (U/L) & $91(70.62-141.78)$ & $78(-42.55-217.05)$ & 0.201 \\
\hline Bilirubin (mg/dL) & $0.44(0.38-0.67)$ & $0.63(0.18-1.02)$ & 0.082 \\
\hline Peak AST (U/L) & $69.89(63.56-76.21)$ & $186.24(71.84-300.64)$ & $<0.001$ \\
\hline Peak ALT (U/L) & $118.85(103.86-133.85)$ & $153.13(87.82-218.44)$ & 0.91 \\
\hline \multicolumn{4}{|l|}{ Fibrosis scores } \\
\hline AST/ALT & $1.21(1.12-1.30)$ & $1.97(1.41-2.53)$ & $<0.001$ \\
\hline Severe fibrosis depending on AST /ALT $(n, \%)$ & $255(78.7 \%)$ & $36(94.7 \%)$ & 0.02 \\
\hline APRI & $0.61(0.51-0.71)$ & $0.90(0.51-1.29)$ & 0.01 \\
\hline Severe fibrosis depending on APRI $(n, \%)$ & $34(10.5 \%)$ & $7(18.4 \%)$ & 0.14 \\
\hline FIB-4 & $2.60(2.29-2.90)$ & $5.38(3.32-7.44)$ & $<0.001$ \\
\hline
\end{tabular}


Table 3. Cont.

\begin{tabular}{|c|c|c|c|}
\hline Variable & Survivors $(n=327,88.4 \%)$ & Non-Survivors $(n=43,11.6 \%)$ & $p$ \\
\hline Severe fibrosis depending on FIB- $4(n, \%)$ & $162(50.2 \%)$ & $30(78.9 \%)$ & 0.001 \\
\hline Forns & $7.86(7.57-8.15)$ & $9.29(8.60-9.98)$ & $<0.001$ \\
\hline Severe fibrosis depending on Forns $(n, \%)$ & $130(66.7 \%)$ & $30(96.8 \%)$ & $<0.001$ \\
\hline \multicolumn{4}{|l|}{ Disease severity and personal history } \\
\hline Liver steatosis on $\mathrm{CT}$ & $126(38.53 \%)$ & $20(46.51 \%)$ & 0.314 \\
\hline TSS start & $11(10.15-13.35)$ & $12.5(6.62-18.38)$ & 0.004 \\
\hline TSS maxim & $11(10.28-13.72)$ & $12.5(5.77-20.23)$ & $<0.001$ \\
\hline Arterial hypertension & $183(56.5 \%)$ & $28(73.7 \%)$ & 0.04 \\
\hline Ischemic heart disease & $45(13.9 \%)$ & $12(31.6 \%)$ & 0.005 \\
\hline Atrial fibrillation & $30(9.3 \%)$ & $6(15.8 \%)$ & 0.20 \\
\hline Chronic heart failure & $31(9.6 \%)$ & $10(26.3 \%)$ & 0.002 \\
\hline Chronic obstructive pulmonary disease & $23(7.1 \%)$ & $10(26.3 \%)$ & $<0.001$ \\
\hline Stroke & $22(6.8 \%)$ & $3(7.9 \%)$ & 0.79 \\
\hline Chronic kidney disease & $30(9.3 \%)$ & $12(31.6 \%)$ & $<0.001$ \\
\hline Type 2 diabetes mellitus & $75(23.1 \%)$ & $13(34.2 \%)$ & 0.13 \\
\hline Neoplasia & $21(6.5 \%)$ & $5(13.2 \%)$ & 0.13 \\
\hline
\end{tabular}

Abbreviations: ALT-alanine aminotransferase; AST-aspartate aminotransferase; ALP-alkaline phosphatase; GGT—gamma glutamyl transferase; CRP $-\mathrm{C}$ reactive protein; $\mathrm{LDH}-$ lactate dehydrogenase; $\mathrm{TG}-$ triglycerides; LDL—low density lipoproteins; HDL—high density lipoproteins; INR—international normalized ratio; PT—prothrombin time; CT—computer tomography; COPD—Chronic obstructive pulmonary disease; CVA—cerebrovascular accident; T2DM-type 2 diabetes mellitus. Variables are shown as median (95\% confidence interval).

The association between the values of FIB-4, APRI, Forns, AST/ALT exceeding the threshold for a severe score and the presence of liver steatosis was analyzed (Table 4). While severe FIB- 4 was not associated with a higher prevalence of steatosis, high ageadjusted FIB-4 (subgroup of patients over 65 years old, $p=0.01$ ), Forns $(p<0.01)$, APRI $(p=0.03)$ and AST / ALT $(p=0.02)$ scores were more frequently encountered in patients with steatosis. As a matter of nuance, liver enzyme elevations (AST, ALT or both) were more frequently associated with higher scores across the board, suggestive of the presence of fibrosis. However, this relationship was also valid for the Forns score, which does not include AST or ALT in its calculation formula.

We investigated whether the presence of MAFLD (detected via the index CT scan) had any prognostic relevance. No significant associations were found between liver steatosis and COVID-19 severity $(p=0.61)$, hospital stay $(p=0.11)$, ICU admission $(p=0.23)$, or survival $(p=0.56)$.

\subsection{Multivariate Analysis}

All the non-invasive scores were included in a multivariate analysis to assess their predictive value for survival. Among them, only FIB-4 and elevated AST values were significantly associated with poor survival ( $p=0.046$ and $p=0.037$, respectively) (Table 5). Using the conventional cut-off value for high FIB-4 (3.25), the Kaplan-Meyer survival analysis revealed that an FIB-4 exceeding the threshold was associated with a worse survival $(p=0.05)$ (Figure 1). A similar correlation was found for age-adjusted FIB-4 $(p=0.036)$ and Forns $(p<0.001)$. 
Table 4. Association between fibrosis severity, steatosis, and markers of liver injury.

\begin{tabular}{ccc}
\hline Fibrosis Scores & Associations & $p$ \\
\hline \multirow{2}{*}{ Severe FIB-4 } & Steatosis & 0.721 \\
\cline { 2 - 3 } & ALT $>50$ & 0.196 \\
\cline { 2 - 3 } & AST $>50$ & $<0.001$ \\
\cline { 2 - 3 } Severe FIB-4 $>65$ & Steatosis & 0.012 \\
\cline { 2 - 3 } & ALT $>50$ & $<0.001$ \\
\cline { 2 - 3 } & AST $>50$ & 0.008 \\
\cline { 2 - 3 } Severe Forns & Steatosis & 0.001 \\
\hline Severe APRI & ALT $>50$ & 0.001 \\
\hline AST / ALT & AST $>50$ & 0.001 \\
\hline
\end{tabular}

Abbreviations: ALT—alanine aminotransferase; AST—aspartate aminotransferase.

Table 5. Multivariate analysis of fibrosis scores for survival prediction.

\begin{tabular}{cccc}
\hline Fibrosis Scores & OR & Std Error & $p$ \\
\hline FIB-4 & 1.353 & 0.151 & 0.046 \\
\hline AST $>50$ & 1.02 & 0.009 & 0.037 \\
\hline AST $/$ ALT & 1.46 & 0.262 & 0.144 \\
\hline APRI & 0.21 & 0.985 & 0.119 \\
\hline Forns & 1.131 & 0.144 & 0.391
\end{tabular}

Abbreviations: ALT—alanine aminotransferase; AST- aspartate aminotransferase; OR—odds ratio; Std errorstandard error.

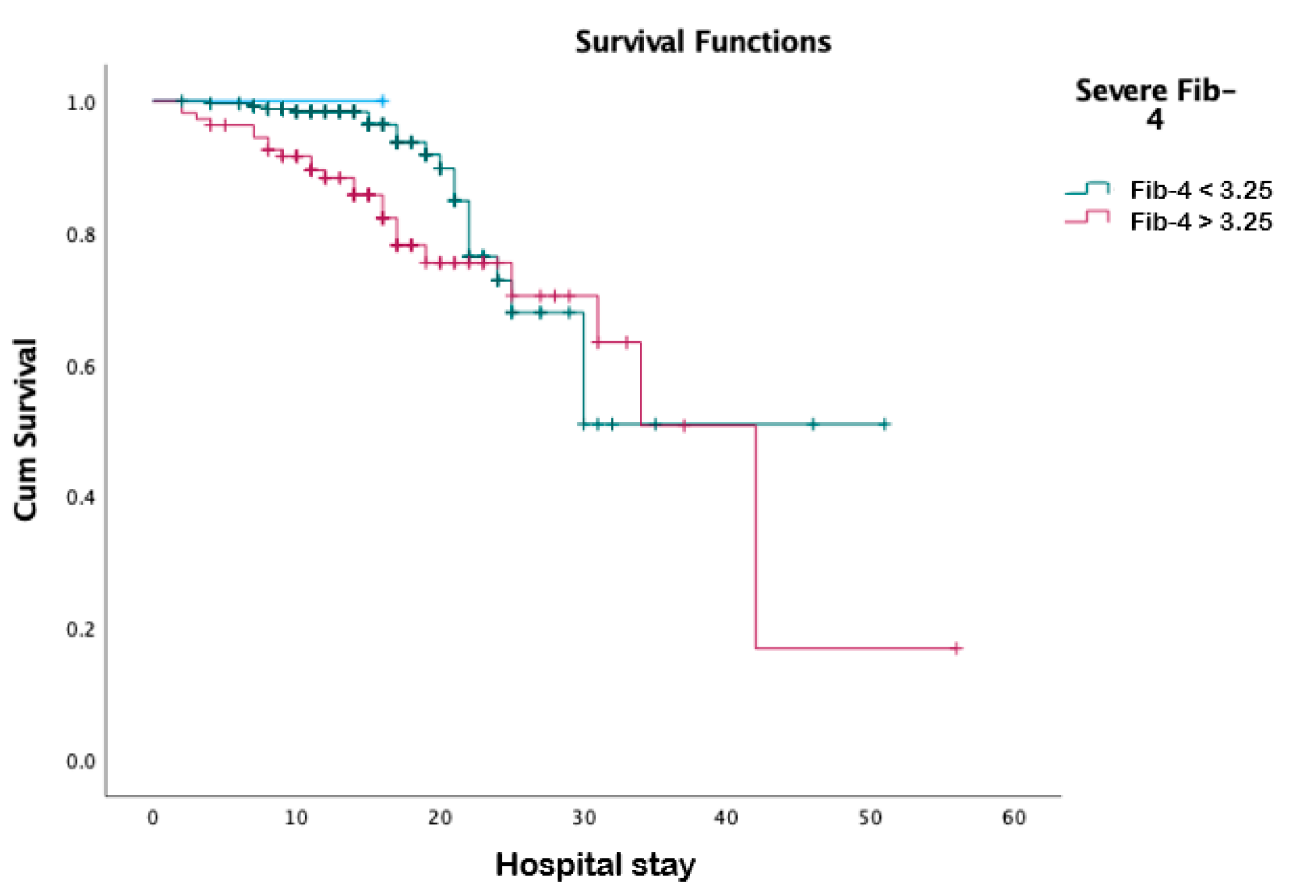

Figure 1. Survival analysis for severe FIB-4 score, defined as FIB-4 > 3.25.

We also analyzed the AUROC curves for the fibrosis scores (Figure 2) and found that FIB-4 had the best predictive value for survival, with an area under the curve (AUC) of 
0.731, while AST / ALT was a close second, with an AUC of 0.72, both suggestive of good discriminatory performance. The Forns score had an AUC of 0.679 , while APRI had the lowest AUC of 0.622 .

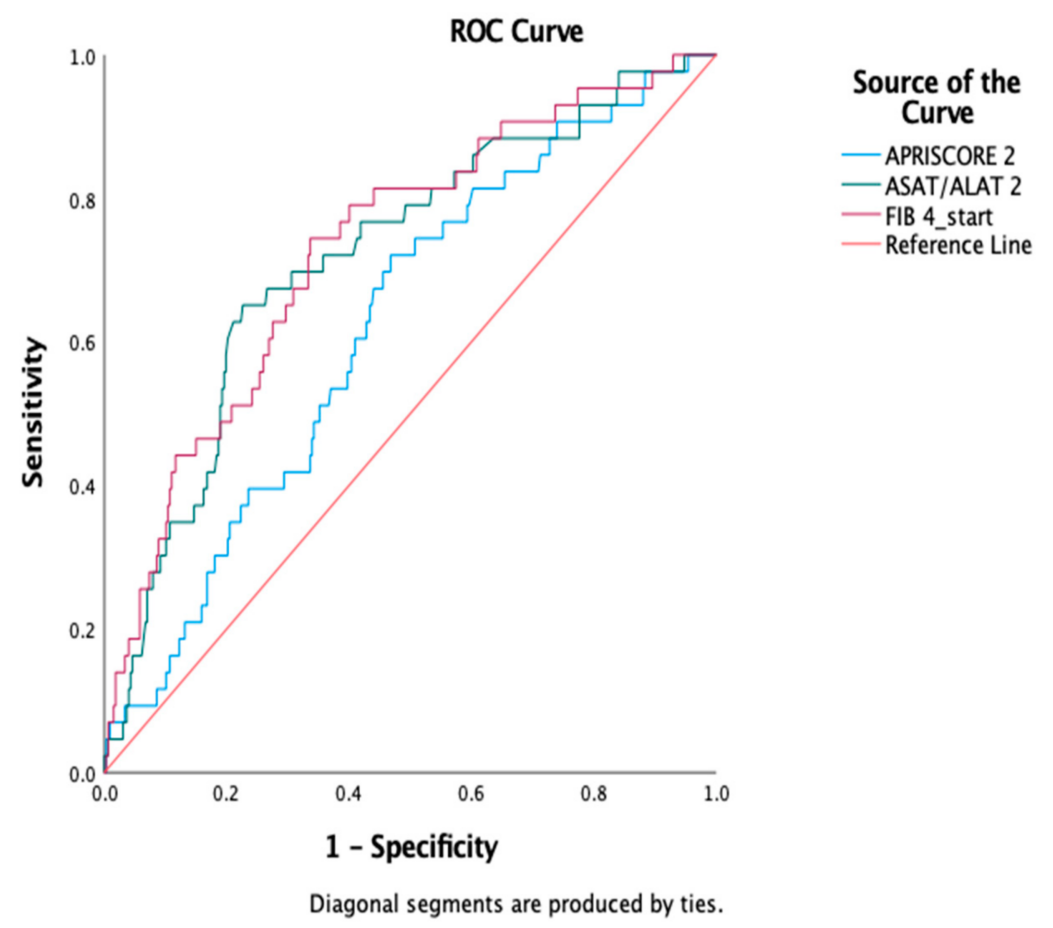

Figure 2. AUROC curves comparison for FIB-4, AST/ALT, APRI scores.

\section{Discussion}

In response to our major objective, the results suggest that abnormal liver enzyme levels and high non-invasive scores in assessing the risk of advanced fibrosis are associated with a worse in-hospital outcome. We found a very high prevalence of abnormal liver biochemistry tests. Over two-thirds of our cohort $(>72 \%)$ had elevated transaminases, while more than half of the patients (50.92\%) also had cholestasis. These figures are significantly higher than previously published reports, which place the prevalence of liver test abnormalities between 14 and 53\% [8]. However, given the nature of our design, asymptomatic patients or those on the less severe end of the disease spectrum were not included in our cohort. Therefore, the balance was skewed towards moderate and severe forms, with a higher prevalence of systemic injury. Hepatic involvement is thought to be related to direct viral infection of the liver cells, sepsis, or drug hepatotoxicity [24]. Since patients presented with high AST and ALT values at admission in our cohort, drug hepatotoxicity seems unlikely. Emerging data have shown that the SARS-CoV-2 virus can have a direct cytopathic effect on hepatocytes and cholangiocytes, with autopsy reports detecting viral material in the liver tissue in up to $41 \%$ of cases. The histological pattern is unspecific, appearing to be similar to drug-induced liver injury or sepsis. The immune response can also be dysregulated, leading to the activation of cytokines and subsequent hepatic inflammation [25]. The high number of patients that presented with elevated liver enzymes might therefore be indicative of the direct infection of the liver cells. However, the relevance of incidental findings of elevated transaminases or cholestasis on disease outcome is to this point unclear, as the liver might be only a quiescent collateral victim of systemic inflammation and viral ubiquity in the absence of overt liver failure. Our study found no statistical difference between liver enzyme values at admission between survivors and non-survivors. On the other hand, peak levels of AST were significantly higher in non-survivors, suggesting that liver injury might be associated with the risk of death, which is in concordance with other studies [26]. 
Preliminary reports have additionally proved that pre-existing liver diseases lead to worse outcomes for COVID-19 patients [27-29]. However, in our group, we excluded patients with previously diagnosed liver diseases and screened for the most common causes for hepatic injury, by testing for hepatitis B and C. However, the impact of subclinical or unrecognized liver disease in COVID-19 patients is still unknown, and the most common culprit is MAFLD. In this light, a simple, reliable score that could help clinicians categorize patients who need further assessment may be of great help in planning a subsequent liver-related work-up.

There is a wide range of non-invasive tests designed to rule in or out chronic liver disease and, more specifically, the presence of advanced fibrosis. Their perks and pitfalls have been elegantly addressed in the most recent EASL position paper on the topic, published in the summer of 2021 (quote in commentary). Nevertheless, beyond their well-defined indications in the setting of previously documented liver disease or at-risk liver disease populations, the role of these non-invasive tests has been tested in more unorthodox scenarios. Their use has not necessarily been as indicators of advanced fibrosis, but rather as predictive factors for liver-related outcomes. In this framework, recent studies have suggested that FIB-4 could have a potential prognostic role in COVID-19 [11,14,30,31]. Our study further augments this idea since in our group of patients, presumably without any underlying liver disease, this score was significantly associated with the risk of death, with an AUROC of 0.74, the highest compared to other liver fibrosis scores. Furthermore, besides a high AST value, FIB-4 levels were the only ones that retained statistical significance in the multivariate analysis $(p=0.05)$. We opted for FIB- 4 because of its simplicity and high validation in different liver etiologies [32]. We also applied other fibrosis scores, such as AST/ALT, APRI, and Forns, which had a limited predictive power.

As expected, high non-invasive scores (FIB-4 $>65$, Forns and APRI) exceeding the cut-offs for advanced fibrosis, were all correlated with steatosis. Furthermore, high AST and ALT values (>50 UI/1), were also significantly associated with severe FIB-4 $>65$ and Forns. While the association between FIB- 4 and high transaminase levels was expected since the calculation formula includes AST and ALT (which are frequently elevated in coronavirus patients), their correlation with the Forns score was not a given. This key aspect might support the assumption that liver injury expands beyond incidental laboratory findings of elevated liver enzymes that can artificially alter non-invasive scores above conventional cut-offs, although the exact mechanisms are poorly defined. As proof, the Forns score was also statistically associated with survival prediction using the Kaplan-Meyer estimator $(p<0.001)$, which raises the question of whether non-invasive fibrosis evaluation should be performed for every patient, given their prognostic role in COVID-19.

The reason why some fibrosis scores' elevation, mainly FIB-4, is related to COVID-19 outcome is still unclear. Samaniego et al. [11] pointed out the prognostic role of FIB-4 in 160 patients diagnosed with SARS-CoV-2 infection and proceeded to calculate the FIB-4 score in a subgroup (15\% of the total), retrieving liver tests performed within six months of the diagnosis. They discovered that while AST and ALT levels were significantly higher at the time of COVID-19 infection, levels of FIB-4 were not significantly different. They concluded that high FIB-4 (>2.67) as a marker for advanced liver fibrosis could have a prognostic role for coronavirus patients. Their explanations reside from the fact that chronic liver disease is associated with a degree of baseline systemic inflammation that adds to COVID-19's inflammatory response. Our study further nuances the matter of fibrosis scores being elevated. As mentioned above, a high FIB-4 could be explained by high levels of liver enzymes, which might be a result of cytopathic virus-related liver injury or systemic inflammation. On the other hand, other scores, like Forns, are not calculated using transaminase values, yet they still reach levels compatible with advanced fibrosis. This prompts a further question: are these scores truly indicative of histological fibrosis or do they reflect a type of liver injury associated with a worse outcome through other means? Their validation and role should be further explored in larger cohorts and additionally 
correlated with elastography tests. Unfortunately, in the context of COVID-19, these designs are difficult to implement, especially in high-disease-burden settings.

Another study on 70 patients with different hematological malignancies showed that FIB-4 $>3.85$ was an independent predictor for mortality in patients with COVID-19 infection; the authors point out that the synergistic effect of SARS-CoV-2 virus on the liver and intrinsic hematopoietic abnormalities found in this subcategory of patients led to higher FIB-4 values, although admittedly their sample size was small [33]. Furthermore, a meta-analysis revealed that high FIB-4 values are associated with mortality in COVID-19 patients, thus augmenting the idea that this simple score used for detection of liver fibrosis and can be repurposed for predicting clinical outcome in COVID patients [32].

Globally, MAFLD prevalence is estimated at $24 \%$ of the population [34] and in COVID-19 patients, its prevalence is approximately 30\% [35]. In our study, 146 (39.5\%) patients had steatosis, diagnosed through CT imaging. The relatively higher percentage might be explained by the inclusion of patients with a more severe form of COVID-19 who required hospitalization, therefore who had a higher burden of co-morbid conditions associated with a metabolic imbalance and, consequently, MAFLD.

Studies that have evaluated MAFLD patients have related this disease to poor COVID-19 outcomes [36,37]. The exact mechanism is still unknown, although the proposed hypothesis revolves around the idea that steatosis leads to increased production of proinflammatory cytokines, thus exacerbating the cytokine storm related to SARS-CoV-2 infection $[38,39]$. Furthermore, MAFLD patients with high NFS (NAFLD fibrosis score) and high FIB-4 were associated with a higher risk of severe COVID-19 disease, even after adjusting for obesity and diabetes [40]. Not least, there is purported evidence of a high susceptibility to numerous infections, which typically follow a more severe disease course when compared to non-MAFLD patients. The implications range from recurrent and more severe bacterial pneumonia, urinary tract infections and urolithiasis, Clostridoides difficile colitis, and even more frequent and severe complications of Helicobacter pylori infection [18]. Our study assessed the impact of imaging diagnosed MAFLD disease on hospital stay, risk of death, and severity of the disease (according to TSS score). However, no statistically significant associations were found. The results comply with another study on a cohort of 539 patients that did not find a positive association between MAFLD and disease severity, mortality, or risk of progression [21]. However, some of these studies were limited by the fact that they used the HSI (hepatic steatosis index) score, which could have overestimated the presence of MAFLD [2]. Jin D. et al. pointed out that these conflicting results are additionally influenced by the different methods of establishing COVID-19 severity: either based on the need for mechanical ventilation, ICU admission, or on the premise that patients who did not need oxygen supplementation and could safely be home managed do not represent severe cases. The strength of our study resides in the fact that we established a diagnosis of steatosis based on CT evaluation, which, as mentioned, many of the studies involving either MAFLD or fibrosis scores lack. Additionally, we excluded the most common viral diseases (hepatitis B and C) as the underlying causes of previous liver disease.

We acknowledge that our study has multiple limitations, some of which are the direct result of conducting a clinical study within the barriers of a COVID-19 hospital setting. One such limitation was using fibrosis scores without histological assessment for direct comparison. However, we believe that assessing liver histology through liver biopsy in the context of COVID-19 was not a primary objective and might have harbored little clinical relevance for the purpose of our study. Furthermore, our cohort size was relatively modest. With regards to the use of non-invasive scores, there is not yet an established cut-off point for defining high FIB-4 values in the COVID-19 population. We used the 3.25 cut-off, typically utilized to rule out advanced fibrosis in high-risk patients with MAFLD, given the relatively high burden of steatosis in our cohort, in spite of some studies using other values. We decided against calculating our own cut-off, considering that it would have further complicated the already heterogeneous literature on the topic. Another important issue 
regards the reason for this particular score to be elevated, since in our group of patients, without any liver diseases and regardless of steatosis, it was still associated with the risk of death. Large cohort studies demonstrated that fibrosis scores such as FIB-4 also have limitations in the general population [41] so our result must be interpreted in the COVID19 clinical scenario. However, in this context, FIB-4 may be elevated and considered a predictor for mortality regardless of any subclinical or undiagnosed liver disease. Further research that explains the pathogenic mechanism behind the correlation of high FIB-4 levels and risk of death in COVID-19 patients is needed.

\section{Conclusions}

Given the impact of the ongoing pandemic on the healthcare system and the expected bleak effect from deferring or cancelling treatments for chronic illnesses and delaying diagnosis for acute conditions, every datum on developing a rapid diagnosis for patients is crucial. Whether it is about recognizing an undiagnosed liver disease or predicting a severe outcome for COVID-19 patients, we demonstrated that fibrosis scores like FIB-4 could aid clinicians to categorize patients regarding prognosis and approach them correspondingly, and maybe even prioritize them for vaccination since they represent a group with a worse prognosis.

Author Contributions: Conceptualization D.C., L.A. and R.C., Methodology D.C. and R.C., Formal analysis D.C., Investigation D.C., L.A., C.G. and R.C., Data curation D.C., D.R., S.C., A.G. and V.M., writing-original draft preparation D.C., L.A., C.G., D.R., E.B., L.S., A.M., A.D., B.M., V.D. and R.C., writing-review and editing D.C., L.A., C.G., A.D., D.R., S.C., A.G., V.M., E.B., L.S., L.R., F.C., D.B.J., O.M., L.M., M.R., A.N. and R.C., Supervision D.C. and R.C. All authors have read and agreed to the published version of the manuscript.

Funding: This research received no external funding.

Institutional Review Board Statement: The study was conducted according to the guidelines of the Declaration of Helsinki and approved by the Institutional Ethics Committee of the Clinical Municipal Hospital Cluj-Napoca.

Informed Consent Statement: Informed consent was obtained from all subjects involved in the study.

Conflicts of Interest: The authors declare no conflict of interest.

\section{References}

1. Fajgenbaum, D.C.; June, C.H. Cytokine Storm. N. Engl. J. Med. 2020, 383, 2255-2273. [CrossRef]

2. Nishiga, M.; Wang, D.W.; Han, Y.; Lewis, D.B.; Wu, J.C. COVID-19 and cardiovascular disease: From basic mechanisms to clinical perspectives. Nat. Rev. Cardiol. 2020, 17, 543-558. [CrossRef] [PubMed]

3. Feldman, E.L.; Savelieff, M.G.; Hayek, S.S.; Pennathur, S.; Kretzler, M.; Pop-Busui, R. COVID-19 and Diabetes: A Collision and Collusion of Two Diseases. Diabetes 2020, 69, 2549-2565. [CrossRef]

4. Stefan, N.; Birkenfeld, A.L.; Schulze, M.B.; Ludwig, D.S. Obesity and impaired metabolic health in patients with COVID-19. Nat. Rev. Endocrinol. 2020, 16, 341-342. [CrossRef] [PubMed]

5. $\quad$ Fung, M.; Nambiar, A.; Pandey, S.; Aldrich, J.M.; Teraoka, J.; Freise, C.; Roberts, J.; Chandran, S.; Hays, S.R.; Bainbridge, E.; et al. Treatment of immunocompromised COVID-19 patients with convalescent plasma. Transpl. Infect. Dis. 2021, 23, e13477. [CrossRef] [PubMed]

6. Brenner, E.J.; Ungaro, R.C.; Gearry, R.B.; Kaplan, G.G.; Kissous-Hunt, M.; Lewis, J.D.; Ng, S.C.; Rahier, J.-F.; Reinisch, W.; Ruemmele, F.M.; et al. Corticosteroids, But Not TNF Antagonists, Are Associated with Adverse COVID-19 Outcomes in Patients with Inflammatory Bowel Diseases: Results from an International Registry. Gastroenterology 2020, 159, 481-491. [CrossRef] [PubMed]

7. Tian, Y.; Qiu, X.; Wang, C.; Zhao, J.; Jiang, X.; Niu, W.; Huang, J.; Zhang, F. Cancer associates with risk and severe events of COVID -19: A systematic review and meta-analysis. Int. J. Cancer 2021, 148, 363-374. [CrossRef] [PubMed]

8. Jothimani, D.; Venugopal, R.; Abedin, M.F.; Kaliamoorthy, I.; Rela, M. COVID-19 and the liver. J. Hepatol. 2020, 73, 1231-1240. [CrossRef] [PubMed]

9. Sterling, R.K.; Lissen, E.; Clumeck, N.; Sola, R.; Correa, M.C.; Montaner, J.; Sulkowski, M.S.; Torriani, F.J.; Dieterich, D.T.; Thomas, D.L.; et al. Development of a simple noninvasive index to predict significant fibrosis in patients with HIV/HCV coinfection. Hepatology 2006, 43, 1317-1325. [CrossRef] [PubMed] 
10. Vilar-Gomez, E.; Chalasani, N. Non-invasive assessment of non-alcoholic fatty liver disease: Clinical prediction rules and blood-based biomarkers. J. Hepatol. 2018, 68, 305-315. [CrossRef]

11. Ibáñez-Samaniego, L.; Bighelli, F.; Usón, C.; Caravaca, C.; Carrillo, C.F.; Romero, M.; Barreales, M.; Perelló, C.; Madejón, A.; Marcos, A.C.; et al. Elevation of Liver Fibrosis Index FIB-4 Is Associated with Poor Clinical Outcomes in Patients With COVID-19. J. Infect. Dis. 2020, 222, 726-733. [CrossRef] [PubMed]

12. Romero-Cristóbal, M.; Clemente-Sánchez, A.; Piñeiro, P.; Cedeño, J.; Rayón, L.; del Río, J.; Ramos, C.; Hernández, D.-A.; Cova, M.; Caballero, A.; et al. Possible unrecognised liver injury is associated with mortality in critically ill COVID-19 patients. Ther. Adv. Gastroenterol. 2021, 14, 17562848211023410. [CrossRef]

13. Campos-Murguía, A.; Román-Calleja, B.M.; Toledo-Coronado, I.V.; González-Regueiro, J.A.; Solís-Ortega, A.A.; Kúsulas-Delint, D.; Cruz-Contreras, M.; Cruz-Yedra, N.; Cubero, F.J.; Nevzorova, Y.A.; et al. Liver fibrosis in patients with metabolic associated fatty liver disease is a risk factor for adverse outcomes in COVID-19. Dig. Liver Dis. 2021, 53, 525-533. [CrossRef]

14. Xiang, F.; Sun, J.; Chen, P.-H.; Han, P.; Zheng, H.; Cai, S.; Kirk, G.D. Early Elevation of Fibrosis-4 Liver Fibrosis Score Is Associated with Adverse Outcomes among Patients with Coronavirus Disease 2019. Clin. Infect. Dis. 2021, 73, e594-e601. [CrossRef] [PubMed]

15. Wai, C.-T.; Greenson, J.K.; Fontana, R.J.; Kalbfleisch, J.D.; Marrero, J.A.; Conjeevaram, H.S.; Lok, A.S.-F. A simple noninvasive index can predict both significant fibrosis and cirrhosis in patients with chronic hepatitis C. Hepatology 2003, 38, 518-526. [CrossRef] [PubMed]

16. Lin, Z.-H.; Xin, Y.-N.; Dong, Q.-J.; Wang, Q.; Jiang, X.-J.; Zhan, S.-H.; Sun, Y.; Xuan, S.-Y. Performance of the aspartate aminotransferase-to-platelet ratio index for the staging of hepatitis C-related fibrosis: An updated meta-analysis. Hepatology 2011, 53, 726-736. [CrossRef] [PubMed]

17. Giannini, E.; Risso, D.; Botta, F.; Chiarbonello, B.; Fasoli, A.; Malfatti, F.; Romagnoli, P.; Testa, E.; Ceppa, P.; Testa, R. Validity and Clinical Utility of the Aspartate Aminotransferase-Alanine Aminotransferase Ratio in Assessing Disease Severity and Prognosis in Patients with Hepatitis C Virus-Related Chronic Liver Disease. Arch. Intern. Med. 2003, 163, 218-224. [CrossRef] [PubMed]

18. Adenote, A.; Dumic, I.; Madrid, C.; Barusya, C.; Nordstrom, C.W.; Prada, L.R. NAFLD and Infection, a Nuanced Relationship. Can. J. Gastroenterol. Hepatol. 2021, 2021, 5556354. [CrossRef]

19. Pan, L.; Huang, P.; Xie, X.; Xu, J.; Guo, D.; Jiang, Y. Metabolic associated fatty liver disease increases the severity of COVID-19: A meta-analysis. Dig. Liver Dis. 2020, 53, 153-157. [CrossRef]

20. Mushtaq, K.; Khan, M.U.; Iqbal, F.; Alsoub, D.H.; Chaudhry, H.S.; Ata, F.; Iqbal, P.; Elfert, K.; Balaraju, G.; Almaslamani, M.; et al NAFLD is a predictor of liver injury in COVID-19 hospitalized patients but not of mortality, disease severity on the presentation or progression-The debate continues. J. Hepatol. 2021, 74, 482-484. [CrossRef]

21. Ji, D.; Cheng, G.; Lau, G. Reply to: "NAFLD is a predictor of liver injury in COVID-19 hospitalized patients but not of mortality, disease severity on the presentation or progression-The debate continues. J. Hepatol. 2020, 74, 484-485. [CrossRef]

22. Li, K.; Fang, Y.; Li, W.; Pan, C.; Qin, P.; Zhong, Y.; Liu, X.; Huang, M.; Liao, Y.; Li, S. CT image visual quantitative evaluation and clinical classification of coronavirus disease (COVID-19). Eur. Radiol. 2020, 30, 4407-4416. [CrossRef] [PubMed]

23. McPherson, S.; Hardy, T.; Dufour, J.-F.; Petta, S.; Romero-Gómez, M.; Allison, M.; Oliveira, C.P.; Francque, S.; Van Gaal, L.; Schattenberg, J.M.; et al. Age as a Confounding Factor for the Accurate Non-Invasive Diagnosis of Advanced NAFLD Fibrosis. Am. J. Gastroenterol. 2017, 112, 740-751. [CrossRef] [PubMed]

24. Zhang, C.; Shi, L.; Wang, F.-S. Liver injury in COVID-19: Management and challenges. Lancet Gastroenterol. Hepatol. 2020, 5, 428-430. [CrossRef]

25. Alqahtani, S.; Schattenberg, J.M. Liver injury in COVID-19: The current evidence. United Eur. Gastroenterol. J. 2020, 8, 509-519. [CrossRef]

26. Lei, F.; Liu, Y.; Zhou, F.; Qin, J.; Zhang, P.; Zhu, L.; Zhang, X.; Cai, J.; Lin, L.; Ouyang, S.; et al. Longitudinal Association between Markers of Liver Injury and Mortality in COVID-19 in China. Hepatology 2020, 72, 389-398. [CrossRef] [PubMed]

27. Qi, X.; Liu, Y.; Wang, J.; Fallowfield, J.A.; Wang, J.; Li, X.; Shi, J.; Pan, H.; Zou, S.; Zhang, H.; et al. Clinical course and risk factors for mortality of COVID-19 patients with pre-existing cirrhosis: A multicentre cohort study. Gut 2020, 70, 433-436. [CrossRef] [PubMed]

28. Singh, S.; Khan, A. Clinical Characteristics and Outcomes of Coronavirus Disease 2019 among Patients with Preexisting Liver Disease in the United States: A Multicenter Research Network Study. Gastroenterology 2020, 159, 768-771. [CrossRef]

29. Iavarone, M.; D’Ambrosio, R.; Soria, A.; Triolo, M.; Pugliese, N.; Del Poggio, P.; Perricone, G.; Massironi, S.; Spinetti, A.; Buscarini, E.; et al. High rates of 30-day mortality in patients with cirrhosis and COVID-19. J. Hepatol. 2020, 73, 1063-1071. [CrossRef]

30. Gil Park, J.; Kang, M.K.; Lee, Y.R.; Song, J.E.; Kim, N.Y.; Kweon, Y.O.; Tak, W.Y.; Jang, S.Y.; Lee, C.; Kim, B.S.; et al. Fibrosis-4 index as a predictor for mortality in hospitalised patients with COVID-19: A retrospective multicentre cohort study. BMJ Open 2020, 10, e041989. [CrossRef]

31. Li, Y.; Regan, J.; Fajnzylber, J.; Coxen, K.; Corry, H.; Wong, C.; Rosenthal, A.; Atyeo, C.; Fischinger, S.; Gillespie, E.; et al. Liver Fibrosis Index FIB-4 Is Associated with Mortality in COVID-19. Hepatol. Commun. 2020, 5, 2021. [CrossRef] [PubMed]

32. Pranata, R.; Yonas, E.; Huang, I.; Lim, M.A.; Nasution, S.A.; Kuswardhani, R.A.T. Fibrosis-4 index and mortality in coronavirus disease 2019. Eur. J. Gastroenterol. Hepatol. 2021. [CrossRef] 
33. Sutandyo, N.; Kurniawati, S.A.; Jayusman, A.M.; Syafiyah, A.H.; Pranata, R.; Hanafi, A.R. Repurposing FIB-4 index as a predictor of mortality in patients with hematological malignancies and COVID-19. PLoS ONE 2021, 16, e0257775. [CrossRef]

34. Younossi, Z.M.; Koenig, A.B.; Abdelatif, D.; Fazel, Y.; Henry, L.; Wymer, M. Global epidemiology of nonalcoholic fatty liver disease-Meta-analytic assessment of prevalence, incidence, and outcomes. Hepatology 2016, 64, 73-84. [CrossRef]

35. Zheng, K.; Gao, F.; Wang, X.-B.; Sun, Q.-F.; Pan, K.-H.; Wang, T.-Y.; Ma, H.-L.; Chen, Y.-P.; Liu, W.-Y.; George, J.; et al. Letter to the Editor: Obesity as a risk factor for greater severity of COVID-19 in patients with metabolic associated fatty liver disease. Metabolism 2020, 108, 154244. [CrossRef]

36. Zhou, Y.; Zheng, K.I.; Wang, X.; Sun, Q.; Pan, K.; Wang, T.; Ma, H.; Chen, Y.; George, J.; Zheng, M. Metabolic-associated fatty liver disease is associated with severity of COVID-19. Liver Int. 2020, 40, 2160-2163. [CrossRef]

37. Ji, D.; Qin, E.; Xu, J.; Zhang, D.; Cheng, G.; Wang, Y.; Lau, G. Non-alcoholic fatty liver diseases in patients with COVID-19: A retrospective study. J. Hepatol. 2020, 73, 451-453. [CrossRef]

38. Narayanan, S.; Surette, F.A.; Hahn, Y.S. The Immune Landscape in Nonalcoholic Steatohepatitis. Immune Netw. 2016, 16, 147-158. [CrossRef]

39. Sharma, P.; Kumar, A. Metabolic dysfunction associated fatty liver disease increases risk of severe COVID-19. Diabetes Metab. Syndr. Clin. Res. Rev. 2020, 14, 825-827. [CrossRef]

40. Targher, G.; Mantovani, A.; Byrne, C.D.; Wang, X.-B.; Yan, H.-D.; Sun, Q.-F.; Pan, K.-H.; Zheng, K.I.; Chen, Y.-P.; Eslam, M.; et al Risk of severe illness from COVID-19 in patients with metabolic dysfunction-associated fatty liver disease and increased fibrosis scores. Gut 2020, 69, 1545-1547. [CrossRef]

41. Foschi, F.G.; Domenicali, M.; Giacomoni, P.; Dall'Aglio, A.C.; Conti, F.; Borghi, A.; Bevilacqua, V.; Napoli, L.; Mirici, F.; Cucchetti, A.; et al. Is there an association between commonly employed biomarkers of liver fibrosis and liver stiffness in the general population? Ann. Hepatol. 2020, 19, 380-387. [CrossRef] 